\title{
Index theorem and overlap formalism with naive and minimally doubled fermions*
}

\author{
Taro Kimura $^{\dagger}$ \\ Department of Basic Science, University of Tokyo \\ Mathematical Physics Laboratory, RIKEN \\ E-mail: kimuraddice.c.u-tokyo.ac.jp \\ Michael Creutz \\ Brookhaven National Laboratory \\ E-mail: Creutz@bnl.gov

\section{Tatsuhiro Misumi} \\ Yukawa Institute for Theoretical Physics, Kyoto University \\ E-mail: misumi@yukawa.kyoto-u.ac.jp
}

\begin{abstract}
We present a theoretical foundation for the index theorem in naive and minimally doubled lattice fermions by studying the spectral flow of a Hermitean version of Dirac operators. We utilize the point splitting method to implement flavored mass terms, which play an important role in constructing proper Hermitean operators. We show the spectral flow correctly detects the index of the would-be zero modes which is determined by gauge field topology and the number of species doublers. Using the flavored mass terms, we present new types of overlap fermions from the naive fermion kernels, with a number of flavors that depends on the choice of the mass terms.
\end{abstract}

The XXIX International Symposium on Lattice Field Theory - Lattice 2011

July 10-16, 2011

Squaw Valley, Lake Tahoe, California

*RIKEN-MP-34, YITP-11-87

${ }^{\dagger}$ Speaker.

¥Authored under contract number DE-AC02-98CH10886 with the U.S. Department of Energy. Accordingly, the U.S. Government retains a non-exclusive, royalty-free license to publish or reproduce the published form of this contribution, or allow others to do so, for U.S. Government purposes. 


\section{Introduction}

Ref. [1] recently presented how to identify the would-be zero modes and their chiralities with staggered fermions, and its application to the staggered versions of the overlap and the Wilson fermions. There have been some relating works on this topic so far, e.g. the single taste generalization [2], the numerical efficiency [3] of the staggered overlap and the phase structure and the chiral limit of the staggered Wilson fermion [4].

This approach is applied by the present authors to other fermions with species doublers, i.e. naive and minimally doubled fermions in Ref. [5], which this report is based on. We identify species in naive and minimally doubled fermions as flavors by using the point-splitting method [6], and then define proper flavored-mass terms to extract the index in the spectral flow. We also present new versions of overlap fermions built on these fermion kernels. Especially we construct a single-flavor naive overlap fermion by using a certain flavored-mass term which assigns negative mass to only one of species.

\section{Point-splitting and flavored-mass terms}

We first introduce the point-splitting method to obtain flavored-mass terms for minimally doubled fermions. In this paper we concentrate on the Karsten-Wilczek fermion [7 rather than other formulations, i.e. Boriçi-Creutz fermion [8] and so on (see [9] for review). We consider the following Dirac operator for the $d=4$ minimally doubled fermion in the momentum space,

$$
D_{\mathrm{md}}(p)=i \sum_{k=1}^{3} \gamma_{k} \sin p_{k}+\frac{i \gamma_{4}}{\sin \alpha}\left(\cos \alpha+3-\sum_{\mu=1}^{4} \cos p_{\mu}\right),
$$

with a free parameter $\alpha$. It has only two zeros located at $p=(0,0,0, \pm \alpha)$. These two species are not equivalent since the gamma matrices are differently defined between them as $\gamma_{\mu}^{\prime}=\Gamma^{\dagger} \gamma_{\mu} \Gamma$. In this case the transformation matrix is given by $\Gamma=i \gamma_{4} \gamma_{5}$.

The point splitting identifies these inequivalent species as independent flavors 《6. In this method each flavor field is defined so that the associated fermion propagator includes only a single pole by multiplying factors removing the other pole,

$$
u\left(p-\alpha e_{4}\right)=\frac{1}{2}\left(1+\frac{\sin p_{4}}{\sin \alpha}\right) \psi(p), \quad d\left(p+\alpha e_{4}\right)=\frac{1}{2} \Gamma\left(1-\frac{\sin p_{4}}{\sin \alpha}\right) \psi(p) .
$$

Here we work in the momentum space, but the forms in the position space are also obtained through the Fourier transformation even when the gauge fields are turned on [6]. To regard the two fields as flavors we consider a flavor-multiplet field as following,

$$
\Psi(p)=\left(\begin{array}{l}
u\left(p-\alpha e_{4}\right) \\
d\left(p+\alpha e_{4}\right)
\end{array}\right)
$$

where $e_{\mu}$ stands for the reciprocal vector. We note in this representation of the fermion field $\gamma_{5}$ multiplication is expressed as

$$
\gamma_{5} \psi(p) \longrightarrow\left(\begin{array}{ll}
+\gamma_{5} & \\
& -\gamma_{5}
\end{array}\right) \Psi(p)=\left(\gamma_{5} \otimes \tau_{3}\right) \Psi(p) .
$$



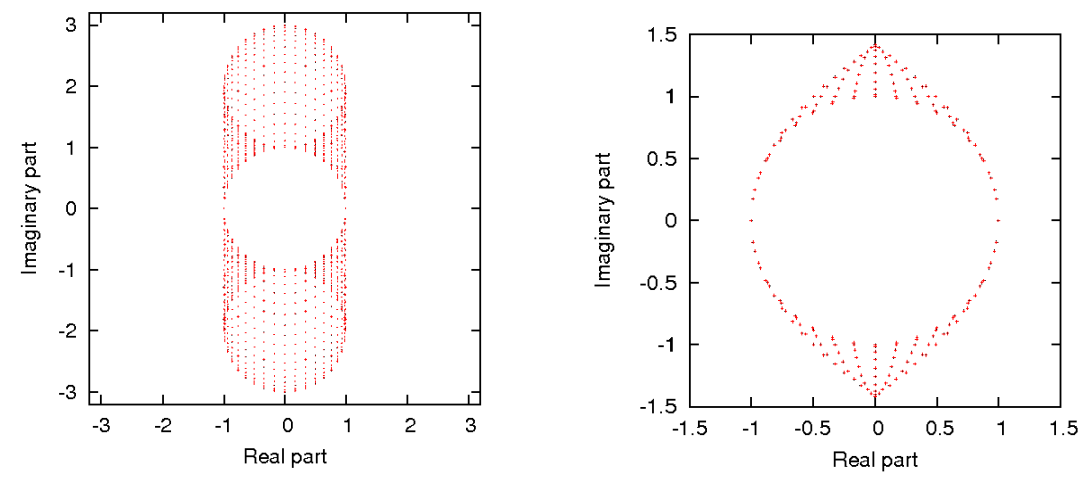

Figure 1: Dirac spectra with flavored mass terms for 2-dimensional free theory: (left) minimally doubled and (right) naive fermions with a mass parameter $m=1$.

This shows the usual $\gamma_{5}$ is a flavor non-singlet gamma-5 as $\gamma_{5} \otimes \tau_{3}$ in the doubler multiplet. Now our purpose is to find a flavored-mass term to assign different masses to the two species. For this purpose we introduce the flavor structure $\tau_{3}$ in the mass term and rewrite it in the usual representation of the fermion field as

$$
\bar{\Psi}(p)\left(\mathbf{1} \otimes \tau_{3}\right) \Psi(p)=\bar{u} u-\bar{d} d=\frac{\sin p_{4}}{\sin \alpha} \bar{\psi}(p) \psi(p) .
$$

In the position space this flavored-mass term is given by

$$
M_{\tau_{3}}=\frac{-i m_{\tau_{3}}}{2 \sin \alpha}\left(T_{+4}-T_{-4}\right) \equiv M_{\mathrm{md}}
$$

with the translation operator $T_{ \pm \mu} \psi_{x}=U_{x, x \pm \mu} \psi_{x \pm \mu}$. Although this flavored-mass term satisfies the gamma-5 hermiticity, in the non-hermitian operator $D_{\mathrm{md}}-M_{\mathrm{md}}$ the kinetic and flavored-mass terms do not commute $\left[D_{\mathrm{md}}, M_{\mathrm{md}}\right] \neq 0$ in the presence of the link variable. It results in the complexvalued Dirac spectrum, which is split into two branches in this case. It indicates the flavored-mass term assigns one of species positive mass and the other negative mass. In Fig. 1 (left) we show a numerical result of complex eigenvalues of the Dirac operator for the $d=2$ free case with a parameter being $\alpha=\pi / 2$.

We then investigate the doubler-multiplet for the naive lattice fermion and the corresponding flavored-mass terms. The Dirac operator for the naive fermion in general dimensions is given by

$$
D_{\text {naive }}(p)=i \sum_{\mu=1}^{d} \gamma_{\mu} \sin p_{\mu} .
$$

For simplicity we here consider the $d=2$ case. It has four zeros, thus we introduce four associated point-splitting fields as

$$
\begin{aligned}
& \psi_{(1)}\left(p-p_{(1)}\right)=\frac{1}{4}\left(1+\cos p_{1}\right)\left(1+\cos p_{2}\right) \Gamma_{(1)} \psi(p), \\
& \psi_{(2)}\left(p-p_{(2)}\right)=\frac{1}{4}\left(1-\cos p_{1}\right)\left(1+\cos p_{2}\right) \Gamma_{(2)} \psi(p), \\
& \psi_{(3)}\left(p-p_{(3)}\right)=\frac{1}{4}\left(1+\cos p_{1}\right)\left(1-\cos p_{2}\right) \Gamma_{(3)} \psi(p), \\
& \psi_{(4)}\left(p-p_{(4)}\right)=\frac{1}{4}\left(1-\cos p_{1}\right)\left(1-\cos p_{2}\right) \Gamma_{(4)} \psi(p),
\end{aligned}
$$




\begin{tabular}{cccc}
\hline \hline label & position & $\chi$ charge & $\Gamma$ \\
\hline 1 & $(0,0)$ & + & $\mathbf{1}$ \\
2 & $(\pi, 0)$ & - & $i \gamma_{1} \gamma_{5}$ \\
3 & $(0, \pi)$ & - & $i \gamma_{2} \gamma_{5}$ \\
4 & $(\pi, \pi)$ & + & $\gamma_{5}$ \\
\hline \hline
\end{tabular}

Table 1: Chiral charges and transformation matrices for each of zeros in the $d=2$ naive fermions with $\gamma_{1}=\sigma_{1}, \gamma_{2}=\sigma_{2}$ and $\gamma_{5}=\sigma_{3}$.

where $p_{i}(i=1,2,3,4)$ stand for the zeros of four species. Their locations of zeros, chiral charges and transformation matrices for the sets of gamma matrices $\gamma_{(i)}^{\mu}=\Gamma_{(i)}^{\dagger} \gamma^{\mu} \Gamma_{(i)}$, are listed in Table 1

The doubler-multiplet field is given by

$$
\Psi(p)=\left(\begin{array}{l}
\psi_{(1)}\left(p-p_{(1)}\right) \\
\psi_{(2)}\left(p-p_{(2)}\right) \\
\psi_{(3)}\left(p-p_{(3)}\right) \\
\psi_{(4)}\left(p-p_{(4)}\right)
\end{array}\right)
$$

and in this case the $\gamma_{5}$ multiplicatioin is expressed as

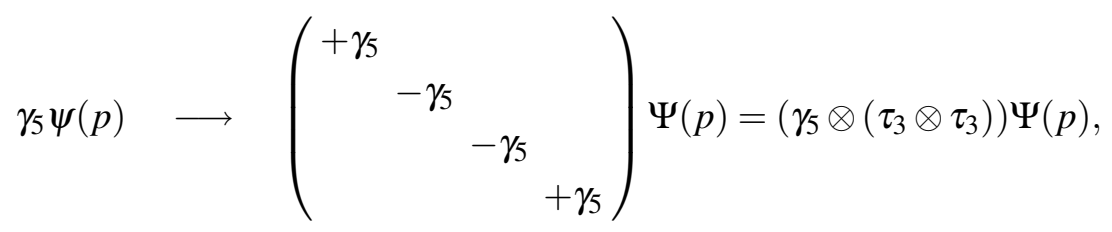

where we express the 4-flavor structure in the doubler-multiplet by two direct products of the Pauli matrix. For our purpose of obtaining the flavored-mass terms to split species, we introduce the following flavor structure in the mass term this time.

$$
\bar{\Psi}(p)\left(\mathbf{1} \otimes\left(\tau_{3} \otimes \tau_{3}\right)\right) \Psi(p)=\cos p_{1} \cos p_{2} \bar{\psi}(p) \psi(p) .
$$

This flavor structure gives two positive and two negative eigenvalues, which implies there are two species with positive and the others with negative mass. Its position-space expression is given by

$$
M_{\tau_{3} \otimes \tau_{3}}=m_{\tau_{3} \otimes \tau_{3}} \sum_{\text {sym. }} C_{1} C_{2} \equiv M_{\text {naive }},
$$

where we define $C_{\mu}=\left(T_{+\mu}+T_{-\mu}\right) / 2$ and $\left(\sum_{\text {sym. }}\right)$ stands for symmetric summation over the order of the factors. In Fig. 1 (right) we show the eigenvalues of the Dirac operator with the flavored mass term, $D_{\text {naive }}-M_{\text {naive }}$. The spectrum is split into two doubled branches crossing the real axis at $|m|$, which indicates the flavored-mass term assigns positive mass to two species while negative mass to the other two.

Furthermore we can consider other kinds of flavored mass terms,

$$
\begin{aligned}
& M_{\tau_{3} \otimes \mathbf{1}}=\frac{1}{2} m_{\tau_{3} \otimes \mathbf{1}} \sum_{\text {sym. }}\left(1+C_{1}^{2}\right) C_{2} \simeq m_{\tau_{3} \otimes \mathbf{1}} C_{2}, \\
& M_{\mathbf{1} \otimes \tau_{3}}=\frac{1}{2} m_{\mathbf{1} \otimes \tau_{3}} \sum_{\text {sym. }} C_{1}\left(1+C_{2}^{2}\right) \simeq m_{\mathbf{1} \otimes \tau_{3}} C_{1} .
\end{aligned}
$$



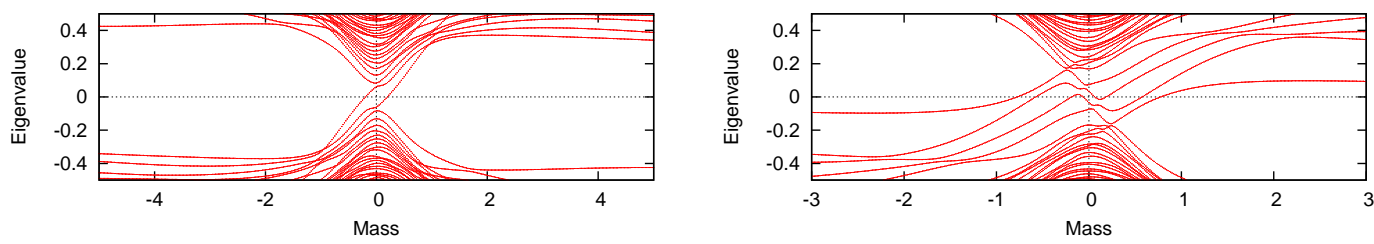

Figure 2: Spectral flows of the Hermitean operator based on the naive fermion for the 2-dimensional case with a topological charge (left) $Q=1$ and (right) $Q=2$.

These terms are utilized to discuss the overlap formalism in section 4 In the $d=4$ case wa can apply the same approach to obtain a proper flavored mass terms [5].

\section{Spectral flow and the index theorem}

In the continuum field theory the index is defined as the difference between the numbers of zero modes of the Dirac operator with positive and negative chirality, $n_{+}$and $n_{-}$. The statement of the index theorem is that the index is just equal to a topological charge $Q$ of a background gauge configuration up to a sign factor depending on its dimensionality,

$$
n_{+}-n_{-}=(-1)^{d / 2} Q
$$

To detect the index of the Dirac operator, it is useful to introduce a certain Hermitean version of the Dirac operator $H(m)=\gamma_{5}(D-m)$, where zero modes of the Dirac operator with \pm chirality correspond to eigenmodes of this Hermitean operator with eigenvalues $\lambda(m)=\mp m$. If we consider the flow of the eigenvalues $\lambda(m)$ as the mass varies, those corresponding to zero modes will cross the origin with slopes $\mp 1$ depending on their chirality. The non-zero eigenmodes of $D$, in contrast, occur in pairs which are mixed by $H$ and cannot cross zero. Therefore the index of the Dirac operator is given by minus the spectral flow of the Hermitean operator, which stands for the net number of eigenvalues crossing the origin, counted with sign depending on the slope.

The index theorem for lattice fermions is also obtained from the spectral flow for not only the Wilson [11], but also the fermions with species doublers [1 [5]. However In the latter cases, the index apparently cancels between pairs of doublers, thus an eigenvalue flow with a simple mass term does not properly capture gauge field topology. This difficulty is resolved by introducing proper flavored-mass terms for staggered [1], minimally doubled and naive fermions [5]. The Hermitean versions of naive and minimally-doubled Dirac operators with flavored-mass terms are

$$
H_{\mathrm{md}}(m)=\gamma_{5}\left(D_{\mathrm{md}}-M_{\mathrm{md}}\right), \quad H_{\text {naive }}(m)=\gamma_{5}\left(D_{\text {naive }}-M_{\text {naive }}\right),
$$

where $M_{\mathrm{md}}$ and $M_{\text {naive }}$ are the flavored-mass terms in (2.6) and (2.15), and $m$ stands for $m_{\tau_{3}}$ for the minimally doubled fermion and $m_{\tau_{3} \otimes \tau_{3}}$ for the naive fermion. Note that the mass terms of these Hermitean operators are flavor-singlet as $\gamma_{5} M_{\mathrm{md}} \simeq \gamma_{5} \otimes \mathbf{1}, \gamma_{5} M_{\text {naive }} \simeq \gamma_{5} \otimes \mathbf{1}$, which is essential in detecting the correct index. Fig. 2] shows the numerical result of the spectral flow for the $d=2$ naive fermion with a topological charge $Q=1$ and $Q=2$, respectively. ${ }^{1}$ There are doubled crossings around the origin, and the number of crossings counted depending on slopes should be the index

\footnotetext{
${ }^{1}$ See $[5$ for details of the numerical simulation and the case with minimally doubled fermion.
} 
related to the topological charge. Taking account of the sign of the slope of the crossings, these results satisfies the index theorem for the naive fermion given by

$$
\operatorname{Index}\left(D_{\text {naive }}\right)=2^{d}(-1)^{d / 2} Q,
$$

where the factor reflects $2^{d}$ species. We can study minimally doubled fermions in a parallel way [5].

\section{Overlap formalism}

The establishment of the index theorem, as discussed in section 3, leads to new versions of overlap fermions [10] based on naive and minimally doubled fermions,

$$
D_{\mathrm{ov}}=1+\gamma_{5} \frac{H(m)}{\sqrt{H(m)^{2}}}
$$

where we now substitute the Hermitean operators defined in (3.2). Here $m$ stands for $m_{\tau_{3}}$ or $m_{\tau_{3} \otimes \tau_{3}}$ again. We note it satisfies the Ginsparg-Wilson relation $\left\{\gamma_{5}, D_{\text {ov }}\right\}=D_{\text {ov }} \gamma_{5} D_{\text {ov }}$. We can obtain the index of the overlap fermion from this Ginsparg-Wilson relation

$$
\operatorname{Index}\left(D_{\text {ov }}\right)=-\frac{1}{2} \operatorname{Tr}\left(\frac{H(m)}{\sqrt{H(m)^{2}}}\right)=-\frac{1}{2} \operatorname{Tr} \operatorname{sgn} H(m) .
$$

Note that the half of the species which have negative mass are converted into physical massless modes in the overlap formulation while the others with positive mass become massive and decouple in the continuum limit. This is because the flavored-mass terms we introduce for minimally doubled and naive fermions assign negative mass to half of species and positive mass to the others. This reduction of flavors also affects the indices of the Dirac operators,

$$
\operatorname{Index}\left(D_{\text {md-ov }}\right)=\frac{1}{2} \operatorname{Index}\left(D_{\text {md }}\right), \quad \operatorname{Index}\left(D_{\text {n-ov }}\right)=\frac{1}{2} \operatorname{Index}\left(D_{\text {naive }}\right) .
$$

This relation relies on the property of the Hermitean operator $\gamma_{5} H(m) \gamma_{5}=-H(-m)$. This is also the case with the staggered overlap, but not the case with the usual Wilson fermion.

We then investigate how to reduce the number of massless modes of the overlap operator with the naive kernel. In the case of the $d=2$ naive fermion with the flavored-mass term (2.15), there are two degenerate species with negative mass, which leads to two overlap massless modes. We lift this degeneracy by adding other kinds of flavored-mass terms in 2.16) and 2.17). To preserve the rotational symmetry we have to consider the following combination,

$$
M_{\text {naive }}(c)=M_{\tau_{3} \otimes \tau_{3}}+c\left(M_{\tau_{3} \otimes \mathbf{1}}+M_{\mathbf{1} \otimes \tau_{3}}\right)
$$

with $m_{\tau_{3} \otimes \tau_{3}}=m_{\tau_{3} \otimes \mathbf{1}}=m_{\mathbf{1} \otimes \tau_{3}}$ in 2.15) 2.16) 2.17). Fig. 3 shows the Dirac spectra and the corresponding spectral flows of the Hermitean operator built with this modified mass term (4.4) for the two $c$ cases. As seen from them, we change the number of negative-mass modes in the original Dirac operator by choosing the parameter $c$. Since such negative-mass modes are converted into massless modes in the overlap formulation, the result in Fig. B indicates a single-flavor overlap fermion is obtained from the naive fermion kernel. The generalization to the $d=4$ is straightforward. In terms of flavored-mass terms the difference between the original overlap and our overlap fermions is just a choice of the flavored mass term. Now we have varieties of overlap fermions including the staggered one and ours, which will be studied further. 

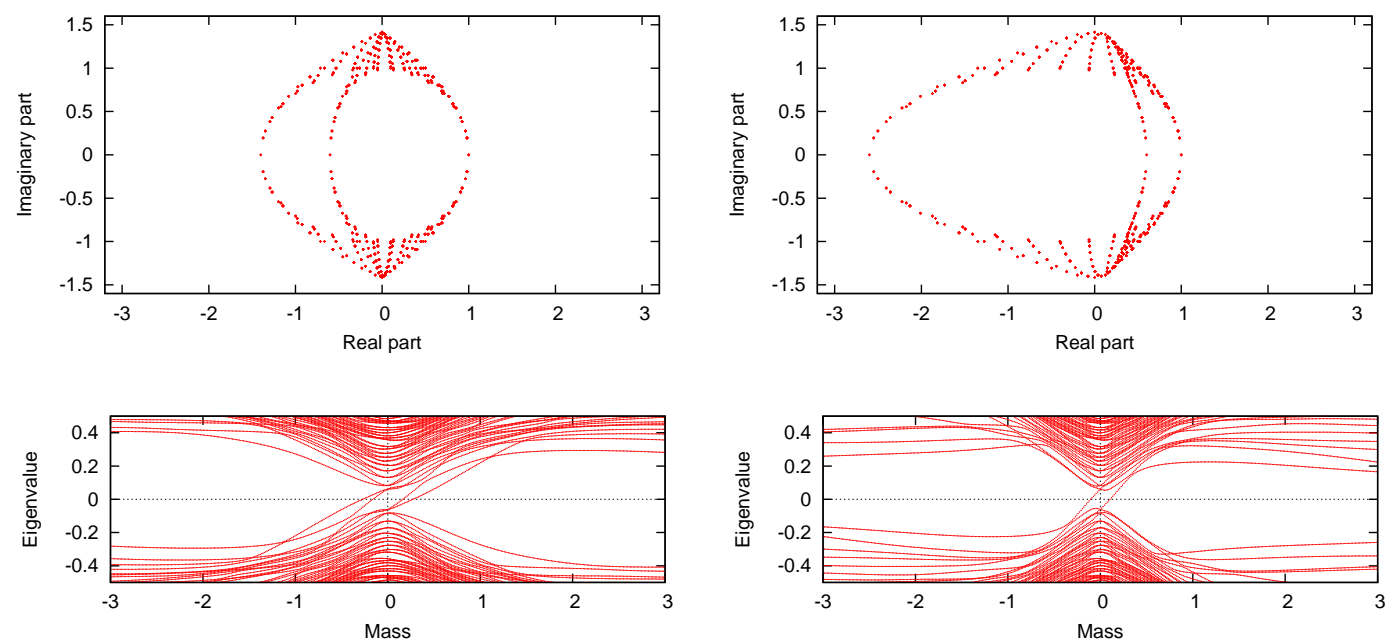

Figure 3: Dirac spectra and the corresponding spectral flows of the naive Hermitean operator with $M_{\text {naive }}(c)$ for (left) $c=0.2$ and (right) $c=0.8$.

\section{Summary}

In this paper we have shown how the index theorem is realized in naive and minimally doubled fermions by considering the spectral flow of the Hermitean version of Dirac operators. The key is to make use of a point splitting for flavored mass terms. We also presented a new version of overlap fermions composed from the naive fermion kernel, which is single-flavored and maintains the hypercubic symmetry essential for a good continuum limit.

\section{References}

[1] D. H. Adams, Phys. Rev. Lett. 104, 141602 (2010) arXiv:0912.2850]; Phys. Lett. B699, 394 (2011) arXiv:1008.2833].

[2] C. Hoelbling, Phys. Lett. B696, 422 (2011) arXiv:1009.5362].

[3] P. de Forcrand, A. Kurkela and M. Panero, PoS: Lattice 2010, 080 (2010) [arXiv:1102.1000].

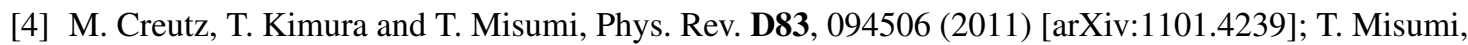
M. Creutz, T. Kimura, T. Z. Nakano and A. Ohnishi, arXiv:1110.1231].

[5] M. Creutz, T. Kimura and T. Misumi, JHEP 1012, 041 (2010) arXiv:1011.0761.

[6] M. Creutz, PoS: Lattice 2010, 078 (2010) [arXiv:1009.3154].

[7] L. H. Karsten, Phys. Lett. B104, 315 (1981); F. Wilczek, Phys. Rev. Lett. 59, 2397 (1987).

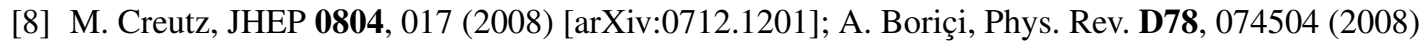
[arXiv:0712.4401].

[9] T. Misumi, M. Creutz and T. Kimura, PoS: Lattice 2010, 260 (2010) [arXiv:1010.3713].

[10] P. H. Ginsparg and K. G. Wilson, Phys. Rev. D25, 2649 (1982); H. Neuberger, Phys. Lett. B427, 353 (1998) hep-lat/9801031.

[11] R. G. Edwards, U. M. Heller and R. Narayanan, Nucl. Phys. B522, 285 (1998) hep-lat/9801015;

D. H. Adams, Annals Phys. 296, 131 (2002) hep-lat/9812003; J. Math. Phys. 42, 5522 (2001) hep-lat/0009026. 